\title{
(6) OPEN ACCESS \\ Development and validation of a simple algorithm for initiation of CPAP in neonates with respiratory distress in Malawi
}

\author{
Shilpa G Hundalani, ${ }^{1,2}$ Rebecca Richards-Kortum, ${ }^{2}$ Maria Oden, ${ }^{2}$ \\ Kondwani Kawaza, ${ }^{1,3}$ Alfred Gest, ${ }^{4}$ Elizabeth Molyneux ${ }^{1,3}$
}

\begin{abstract}
'Department of Pediatrics, Queen Elizabeth Central Hospital, Blantyre, Malawi ${ }^{2}$ Department of

Bioengineering, Rice University, Houston, Texas, USA

${ }^{3}$ University of Malawi, College of Medicine, Blantyre, Malawi ${ }^{4}$ Section of Neonatology, Department of Pediatrics, Baylor College of Medicine, Texas Children's Hospital, Houston, Texas, USA
\end{abstract}

Correspondence to Dr Rebecca Richards-Kortum, Department of Bioengineering, Rice University, MS-142, Houston, TX 77005, USA; rkortum@rice.edu

Received 16 December 2014 Revised 16 March 2015 Accepted 19 March 2015 Published Online First 15 April 2015

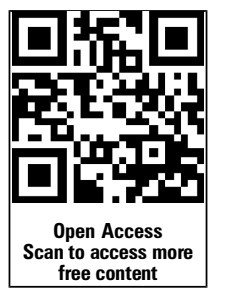

CrossMark

\begin{tabular}{l}
\hline To cite: Hundalani SG, \\
Richards-Kortum R, Oden M, \\
et al. Arch Dis Child Fetal \\
Neonatal Ed 2015;100: \\
F332-F336. \\
\hline
\end{tabular}

To cite: Hundalani SG, et al. Arch Dis Child Fetal Neonatal Ed 2015;100 F332-F336.

\begin{abstract}
Background Low-cost bubble continuous positive airway pressure (bCPAP) systems have been shown to improve survival in neonates with respiratory distress, in developing countries including Malawi. District hospitals in Malawi implementing CPAP requested simple and reliable guidelines to enable healthcare workers with basic skills and minimal training to determine when treatment with CPAP is necessary. We developed and validated TRY ( $T$ : Tone is good, R: Respiratory Distress and $Y=Y e s)$ CPAP, a simple algorithm to identify neonates with respiratory distress who would benefit from CPAP.

Objective To validate the TRY CPAP algorithm for neonates with respiratory distress in a low-resource setting.
\end{abstract}

Methods We constructed an algorithm using a combination of vital signs, tone and birth weight to determine the need for CPAP in neonates with respiratory distress. Neonates admitted to the neonatal ward of Queen Elizabeth Central Hospital, in Blantyre, Malawi, were assessed in a prospective, cross-sectional study. Nurses and paediatricians-in-training assessed neonates to determine whether they required CPAP using the TRY CPAP algorithm. To establish the accuracy of the TRY CPAP algorithm in evaluating the need for CPAP, their assessment was compared with the decision of a neonatologist blinded to the TRY CPAP algorithm findings.

Results 325 neonates were evaluated over a 2-month period; $13 \%$ were deemed to require CPAP by the neonatologist. The inter-rater reliability with the algorithm was 0.90 for nurses and 0.97 for paediatricians-in-training using the neonatologist's assessment as the reference standard.

Conclusions The TRY CPAP algorithm has the potential to be a simple and reliable tool to assist nurses and clinicians in identifying neonates who require treatment with CPAP in low-resource settings.

\section{BACKGROUND}

Respiratory failure is a leading cause of neonatal mortality in the developing world. Respiratory distress can result from complications of preterm birth, pneumonia, sepsis, diarrhoea and intrapartum related causes, which together account for almost $80 \%$ of neonatal deaths globally. ${ }^{1}$ Bubble continuous positive airway pressure (bCPAP) is a safe, effective intervention for spontaneously breathing neonates with respiratory distress. bCPAP is widely used in developed countries and has also

\section{What is already known on this topic?}

- Bubble continuous positive airway pressure (bCPAP) improves neonatal survival.

- Education and training of staff is critical to the success of CPAP.

\section{What this study adds?}

- TRY ( $\mathrm{T}$ : Tone is good, R: Respiratory Distress and $Y=Y e s)$ CPAP algorithm and mnemonic can be a useful tool to triage neonates with respiratory distress in low-resource settings.

- It is simple and easy to use and can be remembered by all clinical staff.

been shown to improve neonatal survival in lowresource settings. ${ }^{2}$ However, barriers exist for the successful wide-scale implementation of bCPAP in the developing world; one such barrier is limited hospital staff experience in using bCPAP and in assessing neonates who could benefit from bCPAP treatment.

A simple and reliable algorithm to determine the need for CPAP is required to triage infants and ensure efficient and effective use of limited resources in developing world hospitals. The use of highly sensitive algorithms to recognise hypoxaemia in developing countries, when accompanied by protocols to administer oxygen to neonates and to children with acute and non-acute lower respiratory tract illnesses, may substantially reduce case fatality. ${ }^{4}$ We developed and validated the TRY CPAP algorithm and mnemonic to help relatively inexperienced healthcare workers correctly identify which neonates should be treated with bCPAP.

\section{METHODS}

Ethics statement

The study protocol was approved by the University of Malawi College of Medicine Research and Ethics Committee (P.05/11/1079) and the Rice University Institutional Review Board (13-102X).

\section{Algorithm and mnemonic}

An algorithm was devised to help nurses and paediatricians-in-training to determine which patients would be administered CPAP (figure 1). 
Figure $1 \operatorname{TRY}(\mathrm{T}$ : Tone is good, $\mathrm{R}$ : Respiratory Distress and $Y=Y e s)$ continuous positive airway pressure (CPAP) mnemonic and algorithm.

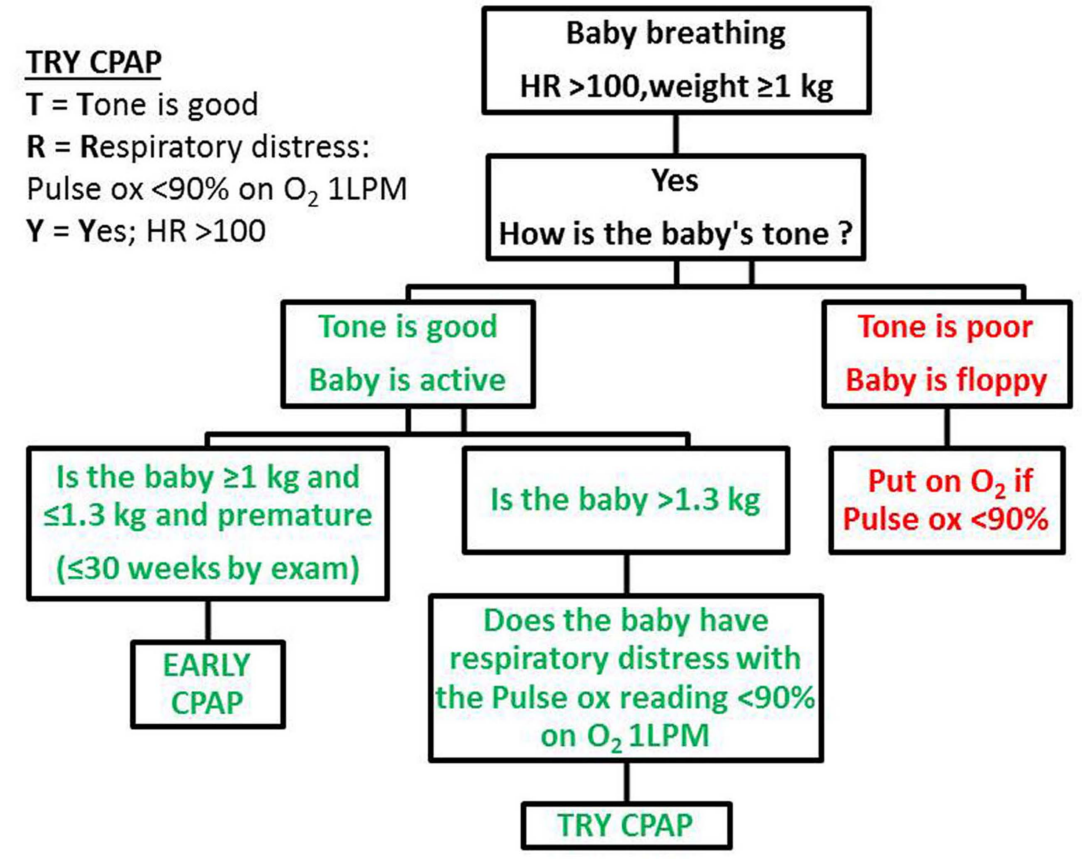

The initial step of the algorithm is to assess whether a spontaneously breathing infant weighing $\geq 1 \mathrm{~kg}$ has a heart rate (HR) over $100 \mathrm{bpm}$. An HR >100 bpm was chosen because if HR is $<100$ after initial resuscitation in the delivery room and presentation to the neonatal ward, the infant would still require active resuscitation $^{5}$ and would have a low APGAR score.

If these criteria are met, the second step of the algorithm is to assess the infant's tone. Tone was assessed in the same manner as in the APGAR score with which nursing and medical staff are familiar. The assessment of tone is subjective and dependent on gestation, but an infant with good tone was defined as one who was moving all four limbs and lay in a flexed posture, whereas a floppy or flaccid infant was one that was not moving, or moving very little and lay with an extended posture. Hypotonia is one of the Sarnat ${ }^{6}$ criteria for severe hypoxic ischaemic encephalopathy (HIE) and was incorporated into the algorithm to avoid putting infants with severe birth asphyxia on CPAP. Infants with severe birth asphyxia are unlikely to benefit from treatment with CPAP and have a $75 \%$ combined probability of severe handicap or death. ${ }^{7}$ Infants with poor tone would receive oxygen by nasal cannulae if pulse oximetry showed an oxygen saturation $\left(\mathrm{SaO}_{2}\right)$ of $<90 \%$.

Infants with tone appropriate for gestational age (GA) are then separated into two groups based on their GA and birth weight. Spontaneously breathing preterm infants $(\leq 1.3 \mathrm{~kg}$; GA $\leq 30$ weeks $)^{8-10}$ with good respiratory effort at birth are treated with early CPAP. The goal of early respiratory management of infants $<30$ weeks GA who are at high risk for respiratory distress syndrome (RDS) is to prevent lung derecruitment, maintain functional residual capacity of the lung, preserve the surfactant monolayer and avoid the cycle of atelectotrauma/volutrauma. Early CPAP helps to achieve these goals and in recent large trials ${ }^{11} 12$ preterm infants born in centres that follow current clinical practices and recommendations ${ }^{13}{ }^{14}$ (greater use of maternal steroids and routine postdelivery stabilisation on CPAP) demonstrate less risk of chronic lung disease and death when stabilised early on CPAP compared with intubation and mechanical ventilation. ${ }^{8}$ Because surfactant and neonatal mechanical ventilators are not available in central and district hospitals in Malawi, early CPAP may help prevent death from RDS in preterm infants. Based on the 2013 Fenton Preterm Growth Chart, the 50th percentile weight for GA in an infant is approximately $1.3 \mathrm{~kg}$ at 30 weeks gestation; we therefore chose this threshold for the weight criterion. ${ }^{15}$ For spontaneously breathing infants $>30$ weeks GA, CPAP is to be initiated if the infant shows signs of respiratory distress and has a $\mathrm{SaO}_{2}<90 \%$ in $\mathrm{O}_{2}$ of $1 \mathrm{~L} / \mathrm{min}$. Many district hospitals in Malawi do not have oxygen flow splitters that will allow for the delivery of $\mathrm{O}_{2}<1 \mathrm{~L} /$ min. Signs of respiratory distress in a neonate are tachypnoea (respiratory rate $>60 / \mathrm{min}$ ), retractions and grunting.

For neonates $>30$ weeks GA at birth, the mnemonic 'TRY' was developed to help nurses and clinical officers remember which patients would benefit from CPAP. TRY stands for: T: Tone is good, R: Respiratory Distress and Y: Yes, HR is $>100 \mathrm{bpm}$.

This prospective cross-sectional study was conducted from mid-July 2014 to mid-September 2014 in the neonatal ward at Queen Elizabeth Central Hospital, in Blantyre, Malawi. Ten nurses or nursing students, six physicians receiving advanced training in paediatrics (registrars, referred to as registrar on call in this paper) and a visiting neonatologist (SH) assessed 325 neonates to determine the appropriate respiratory management. Options included CPAP, $\mathrm{O}_{2}$ by nasal cannulae, or no treatment. On admission of the infant, one nurse or nursing student and one registrar on call determined respiratory management using the TRY CPAP algorithm and this was compared with the clinical assessment of the neonatologist. The clinical assessment of the neonatologist was the reference assessment. They were blind to each other's conclusions. The registrar on call determined whether CPAP was initiated. As we had a limited number of CPAP machines (four in total), in the event that two infants with respiratory distress needed CPAP when only one machine was available, the infant with the higher weight would get preference over the infant with the lower weight. An inter-rater reliability analysis using the $\kappa$ statistic was performed to determine consistency between the assessment of the nurse with that of the neonatologist and the assessment of the registrar with that of the neonatologist.

Spontaneously breathing infants weighing $\geq 1 \mathrm{~kg}$ admitted to the nursery in the first week of life were included in the study. 
Infants with birth weight of $<1 \mathrm{~kg}$, those who arrived dead to the neonatal ward and those with life-limiting congenital anomalies were excluded. Birth weight, vital signs, GA, age at assessment and diagnosis were recorded prospectively from the chart. GA was assessed using the Ballard Score. ${ }^{16}$ Vital signs including $\mathrm{SaO}_{2}$ were measured at admission. $\mathrm{SaO}_{2}$ was measured by placing a fingertip pulse oximeter (Welch Allyn, Nellcor Pulse Oximeter) on the left or right foot of the neonate until the value on the oximeter was constant for at least $10 \mathrm{~s}$. Hypoxaemia was defined as $\mathrm{SaO}_{2}<90 \%$ in room air. Oxygen from oxygen concentrators was always available for children with hypoxaemia.

Midwives initially stabilised infants in the delivery room situated 50 metres from the neonatal ward. Since no CPAP was available in the delivery room or during transport, early CPAP was defined as CPAP initiated on arrival in the neonatal ward.

\section{RESULTS}

Three hundred and twenty-five neonates were enrolled of whom 37 infants (11.4\%) were treated with bCPAP therapy, 15 infants $(4.6 \%)$ received early CPAP therapy and 91 infants (28\%) received oxygen therapy.

The characteristics of the study subjects are shown in table 1. One hundred and fifty-one (46\%) infants of those enrolled were premature (born at GA $<37$ weeks). Eight per cent $(n=26 / 325)$ of our study cohort was $\leq 30$ weeks GA. Median day of life at assessment was the first day of life (range days 1-4). All infants admitted with respiratory distress or presenting with signs suggestive of sepsis, such as temperature instability, hypoglycaemia and jaundice, also received a course of antibiotics to cover for infection. Other infants with maternal risk factors such as preterm labour, maternal fever, prolonged rupture of

\begin{tabular}{|c|c|}
\hline Patient characteristics & $\mathrm{N}=325$ \\
\hline \multicolumn{2}{|l|}{ Gestational age (GA), n (\%) } \\
\hline$<37$ weeks & $151(46.5 \%)$ \\
\hline$\geq 37$ weeks & $174(53.5 \%)$ \\
\hline \multicolumn{2}{|l|}{ Birth weight, n (\%) } \\
\hline $1.0-1.3 \mathrm{~kg}$ & $34(10.5 \%)$ \\
\hline $1.31-2.5 \mathrm{~kg}$ & $159(48.9 \%)$ \\
\hline$>2.5 \mathrm{~kg}$ & $132(40 \%)$ \\
\hline Days of life at assessment, median (IQR) & $0(0-1)$ \\
\hline \multicolumn{2}{|l|}{ Multiple gestation, $n(\%)$} \\
\hline Twins & $37(11.4 \%)$ \\
\hline Triplets & $3(0.9 \%)$ \\
\hline \multicolumn{2}{|l|}{ Admitting diagnosis, n (\%) } \\
\hline Transient tachypnoea of newborn & $78(24 \%)$ \\
\hline Meconium aspiration syndrome & $19(5.9 \%)$ \\
\hline Respiratory distress syndrome & $48(14.8 \%)$ \\
\hline Presumed sepsis & $19(5.8 \%)$ \\
\hline Congenital pneumonia & $7(2.2 \%)$ \\
\hline Haemorrhagic shock & $1(0.3 \%)$ \\
\hline Hypoxic ischaemic encephalopathy & $40(12.3 \%)$ \\
\hline Congenital anomaly & $10(3 \%)$ \\
\hline Hyperbilirubinaemia & $4(1.2 \%)$ \\
\hline $\begin{array}{l}\text { Term low birth weight stable in room air ( } G A>37 \text { weeks, birth } \\
\text { weight }<2.5 \mathrm{~kg} \text { ) }\end{array}$ & $37(11.4 \%)$ \\
\hline $\begin{array}{l}\text { Term macrosomic infants stable in room air (GA>37 weeks, } \\
\text { birth weight } \geq 4 \mathrm{~kg} \text { ) }\end{array}$ & $6(1.9 \%)$ \\
\hline Preterm infants $(\mathrm{GA}<37$ weeks) stable in room air & $56(17.2 \%)$ \\
\hline
\end{tabular}

membranes $>18 \mathrm{~h}$ and multiple vaginal examinations also received antibiotics.

Table 2 shows the number of infants meeting the criteria for CPAP, oxygen or early CPAP using the TRY CPAP algorithm based on the separate assessments of a nurse and a doctor on call compared with the neonatologist's assessment. The neonatologist assessed that 20 neonates (6.2\%) needed early CPAP, 21 (6.5\%) needed CPAP, 87 (26.7\%) qualified for oxygen therapy and 197 (60.6\%) neonates did not require any respiratory support. In comparison, the nurses assessed that $16(5 \%)$ neonates qualified for early CPAP, 18 (5.5\%) required CPAP, 105 $(32.3 \%)$ required oxygen therapy and 186 (57.2\%) did not need any respiratory support. The registrars on call assessed that 15 neonates (4.6\%) qualified for early CPAP, 22 neonates $(6.8 \%)$ qualified for CPAP, 91 neonates $(28 \%)$ needed oxygen therapy and $197(60.6 \%)$ neonates needed no respiratory support. The extra patient put on CPAP by the registrars on call when compared with the neonatologist's assessment was a hypotonic infant with severe HIE that the TRY CPAP algorithm excludes. The differences in assessment of three infants that the nurses determined did not need CPAP when compared with the neonatologist was for one infant with severe sepsis, another with meconium aspiration syndrome (MAS) and a third infant with RDS. For the patient with MAS, the infant's oxygen saturations varied between $88 \%$ and $91 \%$. For the patient with sepsis and RDS, the patient was considered to have depressed tone, and was therefore excluded by the nurses. Taking the neonatologist's assessment as the reference standard, the sensitivity of the algorithm in appropriately deciding the need for CPAP as done by the nurses was $82.9 \%$ (95\% CI $67.9 \%$ to $92.8 \%$ ) and the specificity was $100 \%$ (95\% CI $98.7 \%$ to $100 \%$ ). Similarly, for the registrars on call, the sensitivity and specificity were $87.8 \%$ (95\% CI $73.8 \%$ to $95.9 \%$ ) and $99.6 \%$ (95\% CI $98.0 \%$ to 99.9\%), respectively.

Since the introduction of early postdelivery stabilisation on CPAP of neonates born at $\leq 30$ weeks GA with good respiratory effort at birth was a new concept for the nurses and registrars on call in the neonatal ward, differences in assessments for infants getting early CPAP were expected as part of the learning curve. Thus, two of the four infants whom the nurses assessed as not qualifying for early CPAP and five patients whom the registrars on call did not put on early CPAP were due to failure in recollection of the early CPAP branch of the algorithm. Further, the remaining two of the four infants were excluded by the nurses as they were assessed to be hypotonic.

The inter-rater reliability for the nurses showed a very good agreement with the neonatologist's assessment, $\kappa=0.90,95 \%$ CI (0.86 to 0.95$)$. Similarly, a very good agreement rate was seen for registrars on call with the neonatologist's assessment. ( $=0.97,95 \%$ CI $(0.94$ to 0.99$))$.

\section{DISCUSSION}

This study describes the validation of an algorithm and mnemonic to identify neonates with respiratory distress who would benefit from bCPAP. When compared with the neonatologist's clinical assessment, the algorithm shows very good inter-rater reliability for doctors and nurses.

Mnemonics are frequently used as screening tools in clinical practice or as a guide to clinical management. For example, mnemonic or scoring systems have been employed to identify infants in need of resuscitation, ${ }^{17}$ to describe heart sounds ${ }^{18}$ and to improve critical patient handoffs. ${ }^{19}$ Respiratory scores such as the Silverman Anderson Score and the Downe's RDS score are used to identify respiratory distress in neonates. Each 
Table 2 Comparison of nurse assessment and registrar on call assessment with neonatologist reference assessment using the TRY CPAP algorithm

\begin{tabular}{|c|c|c|c|c|c|}
\hline & \multirow[b]{2}{*}{$n=325$} & \multicolumn{4}{|c|}{ Nurse assessment } \\
\hline & & Early CPAP & CPAP & Oxygen & Room air \\
\hline Neonatologist & Early CPAP & $16(5 \%)$ & 0 & $4(1.2 \%)$ & 0 \\
\hline \multirow[t]{4}{*}{ Reference assessment } & CPAP & 0 & $18(5.5 \%)$ & $3(0.9 \%)$ & 0 \\
\hline & Oxygen & 0 & 0 & $87(26.7 \%)$ & 0 \\
\hline & Room air & 0 & 0 & $11(3.4 \%)$ & $186(57.2 \%)$ \\
\hline & & \multicolumn{4}{|c|}{ Registrar on call assessment and treatment decision } \\
\hline Neonatologist & Early CPAP & $15(4.6 \%)$ & 0 & $5(1.5 \%)$ & 0 \\
\hline \multirow[t]{3}{*}{ Reference assessment } & CPAP & 0 & $21(6.5 \%)$ & 0 & 0 \\
\hline & Oxygen & 0 & $1(0.3 \%)$ & $86(26.5 \%)$ & 0 \\
\hline & Room air & 0 & 0 & 0 & $197(60.6 \%)$ \\
\hline
\end{tabular}

of these scoring systems is based on five parameters and corresponding numerical scores. While these scores are useful to assess severity of respiratory distress, they are hard to remember. We designed the TRY CPAP mnemonic and algorithm to be easy to use and remember. It is based on the essential components of a neonatal examination in order to triage neonates who would benefit from CPAP. For infants $>30$ weeks GA, in a previous study in the same unit, ${ }^{2}$ we found that CPAP was of greatest value to the premature infants with RDS (survival 67\% CPAP vs $23 \%$ nasal Oxygen). Since infants with severe HIE have a combined probability of severe handicap or death of $75 \%,{ }^{7}$ working in limited resource settings with no access to therapeutic hypothermia for infants with HIE, this has helped us to feel more confident that if a choice must be made, to choose to give CPAP to infants with RDS rather than HIE. Early initiation of CPAP in infants $\leq 30$ weeks GA who are at high risk for RDS is currently recommended ${ }^{1314}$ and is thus part of the algorithm. It is also imperative that for best outcomes, along with respiratory care, we also focus on optimal supportive care, including maintenance of normal body temperature, proper fluid management, good nutritional support and support of the circulation to maintain adequate tissue perfusion.

Malawi is one of the few low-income countries that has reached Millennium Development Goal 4 (MDG4) (reduce child mortality by $2 / 3$ from 1990 levels). ${ }^{20}$ The child mortality rate (number of deaths of children under five per 1000 live births) declined to 71 in 2012. If this trend continues, it is expected that by 2015 , the child mortality rate will be 61 , surpassing Malawi's MDG4 target of $78 .^{21}$ Malawi also reduced the infant mortality rate to 46 and the neonatal mortality rate to 24 in $2012 .^{20}$ However, reductions in neonatal deaths have been slow and continue to account for $34 \%$ of deaths of children under five years of age in Malawi. ${ }^{20}$ Twenty-nine per cent of neonatal deaths are due to prematurity; $29 \%$ due to asphyxia; and $26 \%$ are due to infection. ${ }^{22}$ Reductions in neonatal mortality from causes other than tetanus, notably those associated with prematurity and intrapartum complications have been minimal. To reduce neonatal mortality requires increases in coverage as well as systematic attention to high-impact interventions and quality of care. In a study by Buckmaster, ${ }^{23}$ CPAP was shown to be an effective treatment for respiratory distress that significantly improved outcomes in infants born in nontertiary care centres, when it is accompanied by appropriate training and support of medical and nursing staff. As CPAP is implemented in district hospitals in Malawi, efforts to educate and train staff are critical. ${ }^{24}$ Simple tools like the TRY CPAP algorithm can potentially enable inexperienced clinical staff to triage patients appropriately in order to make the best use of available CPAP machines.

The algorithm has some limitations. When using the TRY CPAP algorithm, the staff must be able to distinguish term infants from preterm infants. It is not possible to use only birth weight as the criterion for initiating early CPAP, because there are a number of neonates who are small for GA and weigh $\leq 1.3 \mathrm{~kg}$, but who are more mature and may not need early CPAP. For instance, in our study there were 11 infants with a mean GA of 34 weeks ( $\mathrm{SD} \pm 2$ weeks) with an average birth weight of $1.26 \mathrm{~kg}(\mathrm{SD} \pm 0.08$, range $1.1-1.3 \mathrm{~kg})$. Ten of the 11 neonates were stable in room air and one needed oxygen for a day. There are also infants $>30$ weeks GA struggling to maintain $\mathrm{SaO}_{2}$ in the $90 \%-92 \%$ range and showing other signs of respiratory distress, such as grunting and retractions, who may benefit from early initiation of CPAP. This is especially important for term infants with conditions such as MAS who will benefit from higher oxygen saturations to prevent persistent pulmonary hypertension of the newborn. Continuous monitoring of all infants is imperative for judicious use of limited CPAP resources. Changing the algorithm to initiate CPAP for infants with $\mathrm{SaO}_{2}<95 \%$ as opposed to $<90 \%$ may make it more sensitive in identifying infants with respiratory distress early but at the cost of specificity. In addition, staff should correctly determine infant's tone as appropriate for GA. Assessment of tone should be taught to help in accurate use of the algorithm.

The study itself has some limitations. It is a single-centre study performed in a central hospital in Malawi; the TRY CPAP algorithm has not been tested in the district setting. While the TRY CPAP algorithm still requires validation in a district hospital setting to assess sensitivity, specificity, positive and negative predictive values, and patient variability, this study demonstrates that the TRY CPAP algorithm has the potential to successfully identify neonates who would benefit from CPAP in low-resource settings, where initial treatment decisions may be made by nonphysician clinical staff.

Acknowledgements We would like to thank the nurses and doctors in the neonatal unit at Queen Elizabeth Central Hospital, Blantyre, for their participation and support.

Contributors SGH, RR-K, MO, EM, KK and AG conceptualised and helped design the study. SH completed the data collection, drafted the initial manuscript and carried out the statistical analyses; RR-K, MO, EM, KK and AG coordinated and supervised data collection and critically reviewed and edited the manuscript. All authors had full access to the data and approved the final submission. 
Funding This work is made possible through the generous support of the Saving Lives at Birth partners: The United States Agency for International Development (USAID), the Government of Norway, the Bill and Melinda Gates Foundation, Grand Challenges Canada and the World Bank. It was prepared by the authors and does not necessarily reflect the views of the Saving Lives at Birth partners.

Competing interests None.

Ethics approval University of Malawi College of Medicine Research and Ethics Committee (P.05/11/1079) and the Rice University Institutional Review Board $(13-102 X)$

Provenance and peer review Not commissioned; externally peer reviewed.

Open Access This is an Open Access article distributed in accordance with the Creative Commons Attribution Non Commercial (CC BY-NC 4.0) license, which permits others to distribute, remix, adapt, build upon this work non-commercially, and license their derivative works on different terms, provided the original work is properly cited and the use is non-commercial. See: http://creativecommons.org/ licenses/by-nc/4.0/

\section{REFERENCES}

1 World Health Organization. Global health observatory data repository [website]. Geneva: World health organization, 2014. (accessed 9 Sep 2014).

2 Kawaza K, Machen HE, Brown J, et al. Efficacy of a low-cost bubble CPAP system in treatment of respiratory distress in a neonatal ward in Malawi. PLOS ONE 2014:9:e86327.

3 Martin S, Duke T, Davis P. Efficacy and safety of bubble CPAP in neonatal care in low and middle income countries: a systematic review. Arch Dis Child Fetal Neonatal Ed 2014;99:F495-504.

4 Duke T, Blaschke AJ, Sialis S, et al. Hypoxaemia in acute respiratory and nonrespiratory illnesses in neonates and children in a developing country. Arch Dis Child 2002;86:108-12.

5 Kattwinkel J, Perlman JM, Aziz K, et al. Part 15: Neonatal resuscitation: 2010 American heart association guidelines for cardiopulmonary resuscitation and emergency cardiovascular care. Circulation 2010;122(18 Suppl 3):S909-19.

6 Sarnat HB, Sarnat MS. Neonatal encephalopathy following fetal distress. A clinical and electroencephalographic study. Arch Neurol 1976;33:696-705.

7 Levene MI, Sands C, Grindulis H, et al. Comparison of two methods of predicting outcome in perinatal asphyxia. Lancet 1986;1:67-9.

8 Rojas-Reyes MX, Morley CJ, Soll R. Prophylactic versus selective use of surfactant in preventing morbidity and mortality in preterm infants. Cochrane Database Syst Rev 2012;3:CD000510.
9 Ammari A, Suri M, Milisavljevic V, et al. Variables associated with the early failure of nasal CPAP in very low birth weight infants. J Pediatr 2005; 147:341-7.

10 Schmolzer GM, Kumar M, Pichler G, et al. Non-invasive versus invasive respiratory support in preterm infants at birth: Systematic review and meta-analysis. BMJ 2013:347: 55980.

11 SUPPORT Study Group of the Eunice Kennedy Shriver NICHD Neonatal Research NetworkFiner NN, Carlo WA, et al. Early CPAP versus surfactant in extremely preterm infants. N Engl J Med 2010;362:1970-9.

12 Dunn MS, Kaempf J, de Klerk A, et al. Randomized trial comparing 3 approaches to the initial respiratory management of preterm neonates. Pediatrics 2011;128: e1069-76.

13 Committee on Fetus and Newborn, American Academy of Pediatrics. Respiratory support in preterm infants at birth. Pediatrics 2014;133:171-4.

14 Sweet DG, Carnielli V, Greisen G, et al. European consensus guidelines on the management of neonatal respiratory distress syndrome in preterm infants-2013 update. Neonatology 2013;103:353-68.

15 Fenton TR, Kim JH. A systematic review and meta-analysis to revise the fenton growth chart for preterm infants. BMC Pediatr 2013;13:59.

16 Ballard JL, Novak KK, Driver M. A simplified score for assessment of fetal maturation of newly born infants. J Pediatr 1979;95(5 Pt 1):769-74.

17 Fernandes CJ, Speer ME. Using mnemonics and visual imagery to teach the new neonatal resuscitation program. J Perinatol 2002;22:411-13.

18 Warnica JW. Canadian mnemonics for heart sounds. CMAJ 2007;176:69.

19 McCrory MC, Aboumatar H, Custer JW, et al. "ABC-SBAR" training improves simulated critical patient hand-off by pediatric interns. Pediatr Emerg Care 2012;28:538-43.

20 UNICEF. Committing to child survival: A promise renewed-progress report 2013. 2013.

21 Levels and trends in child mortality report 2013. estimated developed by the UN inter-agency group for child mortality estimation. http://www.childinfo.org/files/ Child_Mortality_Report_2013.pdf (accessed 9 Sep 2014).

22 Requejo J, Bryce J, Victora C. Fulfilling the health agenda for women and children the 2014 report. countdown to 2015 maternal, newborn and child survival. 2014.

23 Buckmaster A. Nasal continuous positive airway pressure for respiratory distress in non-tertiary care centres: What is needed and where to from here? J Paediatr Child Health 2012;48:747-52.

24 Jensen EA, DeMauro SB, Kirpalani $\mathrm{H}$. Has enough evidence accumulated to consider CPAP a first-line standard of care in developing countries? Arch Dis Child Fetal Neonatal Ed 2014;99:F443-4. 